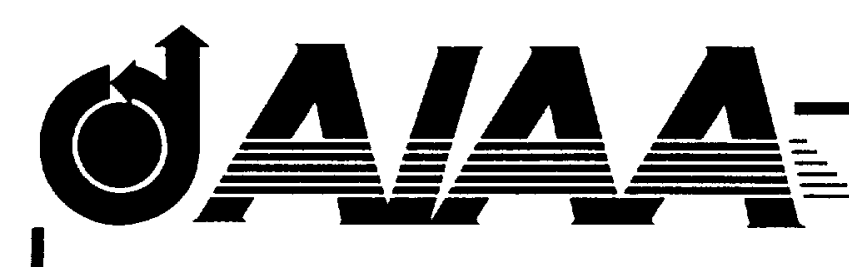

AIAA 2000-2045

\title{
Active Control by Conservation of Energy Concept
}

L. Maestrello

NASA Langley Research Center

Hampton, VA 23681-2199

\section{6th AIAA/CEAS Aeroacoustics Conference 12-14 June 2000 Lahaina, Hawaii}

For permission to copy or republish, contact the American Institute of Aeronautics and Astronautics, 1801 Alexander Bell Drive, Suite 500, Reston, Virginia 20191-4344. 
AIAA-2000-2045

\title{
ACTIVE CONTROL BY CONSERVATION OF ENERGY CONCEPT
}

\author{
Lucio Maestrello \\ NASA Langley Research Center \\ Hampton, VA 23681-2199
}

\begin{abstract}
Three unrelated experiments are discussed; each was extremely sensitive to initial conditions. The initial conditions are the beginnings of the origins of the information that nonlinearity displays. Initial conditions make the phenomenon unstable and unpredictable. With the knowledge of the initial conditions, active control requires far less power than that present in the system response.
\end{abstract}

The first experiment is on the control of shocks from an axisymmetric supersonic jet; the second, control of a nonlinear panel response forced by turbulent boundary layer and sound; the third, control of subharmonic and harmonics of a panel forced by sound. In all three experiments, control is achieved by redistribution of periodic energy response such that the energy is nearly preserved from a previous uncontrolled state. This type of active control improves the performance of the system being controlled.

\section{Introduction}

In science problems, once the sensitivity to initial conditions is observed and the limits of predictability are set, making a quantitative estimate of these limits is possible. Yet the same sensitivity also makes the responses highly susceptible to control. The strategy is to use low-power feedback to redistribute the energy response. Spectra analyses were performed for three unrelated experiments, and their nonlinear behaviors were captured. Active control of the responses for taming irregular dynamics-a change to a lower stable state while maintaining near conservation of energy - was observed.

The dynamics are sensitively dependent on initial conditions; small control adjustments are likely to exert large long-term effects on the response towards a desired control state. The concept of chaos control is very powerful because it offers a variety of possibilities for the dynamic outcome of a particular system response using only tiny adjustments. So far results have been achieved only in the laboratory. The examples described in this paper indicate stabilization of highly unstable periodic orbits under the influence of high intensity noise levels.

The control algorithm has been used in many applications to stabilize an unstable periodic orbit through the application of small, carefully selected perturbations aimed at establishing control over chaotic response. 1,2 This method has since attracted growing interest, and it has been applied in a variety of scientific disciplines. In 1965, Wehrmann ${ }^{3}$ was able to suppress the initial turbulence behind a cylinder in a moving fluid. The basic idea was to vibrate a cylinder with a suitable feedback that used the same fluctuation present in the free shear layer. Control of chaos has been achieved in a variety of physical experiments. ${ }^{4-9}$

Nonlinear time series analysis is becoming a reliable tool for the study of complicated dynamics from measurements. The concept of low-dimensional system response has proven to be fruitful in the understanding of complex problems like elastic structures forced by flow and sound.10-12 Detail examinations $9.13-15$ of the relationship between controllability and chaos have been made that include Lyapunov exponents from the time series using shorttime signal processing. $2,16-18$ These examinations give an indication about the predictability of the dynamics, the dimension of the attractor, and the knowledge of active dynamical degrees of freedom involved in the observation and the initial conditions.

Recent progress in nonlinear dynamical systems has provided the experimentalist with a collection of new tools which have generated a new area of exploration from the measurement of a single time series. 19-21 For example, with an unstable orbit containing a chaotic attractor, it is possible to control the orbit by increasing the symmetry of the observable response and by performing small-amplitude fluctuations about the initial condition, comprising initial time, frequency, phase, and amplitude. The dependence on the initial conditions is known to exist 
in all nonlinear systems, and the time evolution is contingent on an initial typical condition, ' which allows for the control and the manipulation of the chaotic processes. In boundary layer flow, for example, Barenblatt2 I shows that the turbulent energy of the perturbation can either increase monotonically or decrease at first and later start to increase depending on the initial condition. Concern exists about nonlinear and nonsymmetric responses from many different components moving relative to an imposed load that can result in a positive feedback that amplifies the input, destructive interference, or constructive interference, with the former having amplification effects and the latter allowing for deamplification effects. No stationary or time independent state will then be possible.

The understanding of the dynamical system has given the experimentalist a new computer-aided tool to analyze the data. This tool opens new ways for controlling the complex dynamics. Acoustics together with hydrodynamics has been at the forefront of nonlinear dynamics and has supplied the first experimental examples of nonlinearity in acoustics. 22 Three experimental systems are investigated in the present paper, with a view to examining the energy transferred between mode waves to illustrate the role of initial conditions on control strategy using the time series of the measured data.

\section{Results and Interpretation}

Active control of the responses, a change to a lower stable state while maintaining near conservation of energy, was performed in three unrelated experiments. All measurements and control were carried out in real time from direct-current response. 13-15 The first experiment is on control of shocks from an axisymmetric supersonic jet with an oscillating lip; the second, control of a nonlinear panel response forced by an accelerated turbulent boundary layer and sound; the third, control of subharmonic and harmonics of a panel forced by sound.

\subsection{Control of Shocks in a Supersonic Jet}

The pressure from a supersonic jet consists of a shock with spiral and flapping nonaxisymmetric modes superimposed on broadband. Control of the shock at the nozzle lip means either (1) the shock is weakened or is converted into an expansion wave, all or in part, or (2) the vibrating lip attachment prevents the characteristics originating in the column of the shear layer from sustaining connection with an out-of-phase surface vibration. Shock from the jet induces high loading by the fundamental and harmonic tones at different locations on the aircraft structure and annoyance to community residents. Controlling the shock at the nozzle exit has a greater advantage than controlling the aircraft structure.23,24 Powell25 first recognized that the instability of the jet column due to the feedback loop between fluid flow and sound is the cause of a powerful wave moving upstream with sufficient strength to govern the stability of the boundary layer at the nozzle exit. An additional shock discontinuity in the shear layer is located between 9 and 13 nozzle diameters caused by the shear layer transition from supersonic to subsonic as the jet expands. The transitional flow region is an additional source of broadband noise. Later work $26-31$ indicated that selfexcited oscillation involved the transfer of energy from one wave to another, and the spinning of the shock is associated with large-scale instability and sound. Tam 30 presents a theoretical argument to show that the acoustic waves that radiate from the nozzle lip are confined to a relative narrow frequency band if they are generated by the interaction between the large-scale instability waves and the quasi-periodic shock-cell structure of the jet.

A scale model of a round convergent nozzle jet is used in the experiment.13 Time series of the experimental data are used to unravel the complicated dynamics of the shock oscillation. The motion is captured by arrays of pressure transducers and used in the development of a shock-controlling device. Control is achieved by placing a ring at the nozzle lip oscillating at the shock fundamental frequency. This ring prevents the shock characteristics originating in the column of the shear layer from sustaining connection with the outof-phase surface vibration. Shock-free flow is maintained for a large pressure ratio. A convergent nozzle with diameter $D$ of $5.08 \mathrm{~cm}$ is used at a constant pressure ratio of 3 and stagnation temperature of $35^{\circ} \mathrm{C}$, with corresponding Reynolds number $U D / v$ of $1.3 \times 10^{6}$ and Strouhal number $f D / U$ of 0.28 , where $f, U, D$, and $v$ are the shock fundamental frequency, jet flow velocity, nozzle diameter, and kinematic viscosity, respectively. The convergent nozzle with an oscillating ring placed at the lip of the nozzle is shown in Figure 1. This ring is coaxial with the jet and oscillates with the same frequency as the fundamental frequency of the shock. The ring is activated by a feedback loop from the pressure transducer that is placed in the vicinity of the jet exhaust and supplemented by a de bias and a phase filter. ${ }^{3}$ The measured real-time near-field pressures $p(t)$ are shown in Figures 2 and 3 for the uncontrolled and controlled runs, respectively. A remarkable change occurs for the controlled run 
(Figure 3) when compared with the uncontrolled run (Figure 2). The computed power spectral density $P(f, T)$ is shown in Figure 4, where $T$ is the time of the experimental run. For the controlled run, the peak power level $P(f, T)$ is reduced by $40 \mathrm{~dB}$ to broadband level. The peak energy levels of the fundamental and harmonics are being redistributed over the broadband. The integrated levels have nearly the same total power. This conclusion was derived by comparing several experimental runs. 24 The power required to activate the control system is less than 1/200 of the jet acoustical power. The experiment demonstrates that behaviors of shock loading from a supersonic jet can be tamed so that the resulting spectrum response becomes indistinguishable from a typical jet noise spectrum in the absence of shock. Also note that the shock due to shear layer transition from supersonic to subsonic contributes to the broadband noise.

\subsection{Control of Panel Response Forced by Turbulent Boundary Layer and Sound}

Experiments are carried out in a wind tunnel to stabilize and suppress tonal sound and harmonics on a flexible aluminum panel forced by a turbulent boundary layer flow and tonal sound (Figure 5). The structure is mounted on a rigid baffle on a sidewall and consists of two aluminum aircraft-type panels, 65 by 20 by $0.1 \mathrm{~cm}$. The panels deflect toward the moving stream; the mean static deflection is on the order of the panel thickness.

The initial flow velocity is kept small, but as time progresses, the velocity is increased at constant acceleration to a constant terminal velocity of $66 \mathrm{~m} / \mathrm{sec}$. An acoustic source generating $500-\mathrm{Hz}$ pure-tone sound at a power level of $128 \mathrm{~dB}$ is mounted on the opposite sidewall of the tunnel facing the center of the downstream panel. The active controller, an electromagnetic actuator, is mounted at the center of the panel.14 The panel loading and response exhibit convective instability and high-dimensional complex behaviors due to nonlinear coupling between the shortscale instability wave in the boundary layer and the localized intense long scale of the tonal sound and its harmonics. In each run, the panel response exhibits unique dynamics, which indicates a dependency on the initial conditions, that is unpredictable in each run. These regimes can be observed in time series as well as in power spectra. The response $g(t)$ of the panel increases with time as the wind tunnel velocity increases up to a constant flow velocity and is shown in Figure 6 without an external sound and in Figure 7 with pure tone sound superimposed. In each run, the panel response $g(t)$ depends strongly on the forcing amplitude, frequencies, and phase. To suppress the tonal sound, in view of the sensitive dependence on the initial forcing parameters, identifying the initial forcing conditions is necessary to use them as control parameters.? The initial wave remains dominant in the final equilibrium state.

Time series of the wall pressure fluctuations were measured and related to structural response. 14 Measurements of the mean flow velocity profile $u / U$ at constant speed $U$ after the initial start across the turbulent boundary layer $y / L$, where $y$ is the distance across the boundary layer and $L$ is a length scale, are shown in Figure 8 . Significant differences in velocity profiles are indicated when the turbulent boundary layer is forced by pure tone sound compared with the unforced turbulent boundary layer. Repeated runs indicate good repetition for the unforced turbulent boundary layer, whereas for the forced turbulent boundary layer the velocity profile has unrepeatable distributions with variable separation. Results indicate that the nonlinear effect induces large distortion of the mean flows. The kinetic energy of the distorted mean flow is found to be the dominant component of the disturbance kinetic energy. The wall shear stress decreases to local minimum as well as increases to a local maximum. In the outer region, the separation increases to the inviscid free stream in a random fashion.

Active control is applied to the panel motion; the power spectral densities of the acceleration response for the uncontrolled and controlled runs $G(f, T)$ are shown in Figure 9. The uncontrolled run shows a spike at the fundamental forcing frequency of $500 \mathrm{~Hz}$ that exceeds the amplitude of the broadband level by $20 \mathrm{~dB}$ and seven harmonics which are above the broadband. The energy transfer from the fundamental to the harmonics indicates that the forcing acoustic field exceeds a threshold value dominating the broadband. The response is controlled by forcing at the fundamental frequency through a phase amplitude tuning procedure in two stages. In the first stage, the energy of the harmonics is shifted back to the fundamental tone, and in the second stage, the controller reduces the amplitude of the fundamental by adjusting amplitude and phase. Comparing the uncontrolled with controlled spectral density curves shows remarkable changes in the response. The total response power after control varies by a small amount compared with the uncontrolled state; this indicates little energy is lost in the process. The figure also indicates that the energy producing the 
harmonics is supplied by the lower frequencies at about the fundamental frequency. This conclusion has been made after comparing several independent runs.

The response is controlled by a reversing mechanism that generates the high amplitude fundamental and harmonics due to acoustic forcing of the turbulent boundary layer as result of flow-acoustic coupling. The power flow in the structure is carried by the bending wave. As a result of reducing the bending wave response, the structure-born sound will also be reduced. ${ }^{32}$

\section{3. Control of Subharmonic and Harmonics on a Panel Forced by Sound}

Experimental results show that one can convert the nonlinear periodic motion of a panel structure to a linear one by controlling the system about the fundamental orbit through a time-dependent, phaseshift forced perturbation. 15 The experiment was set up in an anechoic chamber, and the panel structure excited by plane acoustic waves at normal incidence. A clamped rectangular aluminum panel 30 by 20 by 0.1 $\mathrm{cm}$ is mounted on a rigid absorbing partition dividing two anechoic rooms, the source, and the transmission rooms (Figure 10). The vibration response is measured by a strain gauge. The change from linear to nonlinear is attributed to the increment of the load at the forcing frequency over a threshold value. Once the nonlinear response is triggered, the oscillations evolve through subharmonic and harmonic formation which may eventually lead to chaos as the acoustic loading increases. The radiated acoustic pressure also contains subharmonics and harmonics. The experiment is designed to demonstrate control of the subharmonic by transferring the energy to the fundamental. The key instability shows an initial bifurcation from a single periodic oscillation to its subharmonic and then harmonic. 33,34 The force is provided by a tonal driving frequency sound $f_{1}$ of period 1 orbit, which becomes unstable as the acoustic loading on the panel increases. As the amplitude of the input force is increased, different dynamic regimes from periodic period 1 to chaos are possible. These regimes can be observed in time series as well as in power spectra and phase portraits. The first period doubling appears at approximately one third of the maximum amplitude. The power spectrum of the strain $S(f, T)$ shows one subharmonic peak at one quarter and two peaks at seven quarters and at the first harmonic of the fundamental, i.e., $f_{1} / 4,7 / 4 f_{1}$, and $2 f_{1}$; the subharmonic is indicated also by the phase response (Figures 11 and 12). The presence of $f_{1} / 4$ in the periodic regime is indicated by the double loop phase plot. The change in response is due to an external controller forcing the panel at the fundamental frequency $f_{1}$. The control run shows that the energy is transferred from the subharmonics and harmonics to the fundamental; as a result, the amplitude of the fundamental increases by the amount of the transfer. Also the double loop phase plot before control merges into a single loop corresponding to a period 1 oscillation of the fundamental control regime. The stabilizing effects change the amplitude of the fundamental; the amplitude increase can be quantitatively estimated in terms of the amplitude of the harmonics and subharmonics and phase angle. The observed changeover to period 1 fundamental while the period 2 subharmonic component was suppressed indicates successful control. The experiment is an example demonstrating energy transfer rather than dissipation during the control process using weak external time-dependent perturbation. Furthermore, damping is unaffected by this control method. Over successive runs, the overall spectra levels of the control and uncontrolled runs maintain nearly the same broadband level.

\section{Discussion}

The basic idea of control is to vary an accessible external parameter in such a way that the system falls into a stable condition at the measured fixed location. We noted that by taming the irregular dynamics-the shock from a jet or the subharmonic on a panel structure-using a small change in the controller parameters has large and rapid effects on response. The transient response changes the energy level; in this case, interference among different wave components leads to constructive and destructive interferences. Each control run manifests different responses due to the different initial conditions; the controller response sets an undesirable state before finally setting the desirable equilibrium condition. Sensitivity to initial conditions can occur with random data. How commonly it occurs has not yet been resolved. We observed and tracked the absolute limits to the minimum amount of dissipation required to maintain stabilization. When the initial condition consists of single frequency, the initial wave remains dominant in final constant input state. When the initial condition consists of two nonharmonic frequencies, the final dominant frequency at the constant input state is the one with the higher initial amplitude. When two 
nonharmonic frequencies have the same initial amplitude, the faster growing wave becomes dominant.

The quasi-nondissipative active control is now discussed. Fluctuations are generally accompanied by dissipation. Stable and unstable periodic responses are very important characteristics of a dynamical system. They provide information about the system phase space response. The periodic response of a system like a vibrating panel is dissipative. However stepping toward manipulating the response using the initial conditions is an appropriate method because the integrated intensity after control is within the integrated intensity prior to control; this indicates a nearly complete energy transfer. Dissipation is present before controlling the response because the orbit of the phase plot is elliptic, a feature attributed to dispersion and damping.

Let us consider the control of a subharmonic (related to the experiment in section 2.3) with a fixed damping coefficient by a small modulation of the driving force of the same frequency. As a simple lumped-parameter system, we model the panel by the well-known Duffing equation, which is a cubic nonlinear oscillator with damping and a harmonic driving force. By assuming a unit mass, the equation takes the form

$$
\ddot{X}+\alpha \dot{X}+\omega_{0}^{2} X+\beta X^{3}=F \cos (\omega t+\phi)
$$

where $\alpha$ is the damping coefficient, $\omega_{0}$ is the natural frequency when $\beta=0$, and $\beta>0$ is a spring constant for the nonlinear restoring force.

From the theory of nonlinear vibration 35 under the appropriate condition on the physical parameters, the nonlinear system can exhibit different responses, such as harmonic response or combined harmonic and subharmonic response. In the case of harmonic response, the approximate solution is given by

$$
X=A \cos \omega t
$$

for which the frequency response curves are determined by the equation:

$$
\left[\left(\omega_{0}^{2}-\omega^{2}\right) A+\frac{3}{4} \beta A^{3}\right]^{2}+\alpha^{2} A \omega^{2}=F^{2}
$$

By varying the forcing amplitude $F$ with all other parameters fixed, the response curves calculated by equation (3) are shown in Figure 13. To seek a subharmonic response, say, of order 3 , the solution of equation (1), 35 is

$$
X=A_{1} \cos \omega t+B_{1} \sin \omega t+A_{1 / 3} \cos \frac{\omega t}{3}
$$

The response relation for harmonic response is similar to Equation (3), and the response relation for the subharmonic is

$$
\omega^{2}=9 \omega_{0}^{2}+\frac{27}{4} \beta\left(A_{1 / 3}^{2}-\frac{F A_{1 / 3}}{8 \omega_{0}^{2}}+\frac{F \cos \phi}{32 \omega_{0}^{4}}\right)
$$

In particular, the condition for the existence of a subharmonic solution of order 3 is

$$
\omega \geq \omega_{1 / 3}=\left[9 \omega_{0}^{2}+\frac{27}{64} \frac{\beta F^{2}}{\omega_{0}^{4}}\left(\frac{\sin ^{2} \phi}{2}-\frac{1}{16}\right)\right]^{1 / 2}
$$

with $\omega=2 \pi f$. In contrast with the harmonic response, the frequency response relations of a subharmonic depend both on the amplitude $F$ and phase $\phi$ of the driving force. This sensitive dependence can be used to control the subharmonic by slight modulation of the phase $\phi$ or amplitude $F$ or both. To illustrate the basic idea, the harmonic response (with $B_{1}=0$ ) and the subharmonic response curves are plotted in the same figure (Figure 14). For instance, at $\omega=\omega_{1 / 3}$, the lowest frequency for the existence of subharmonic, the amplitudes of the subharmonic response and the harmonic response are marked by the points $P$ and $Q$, respectively, in Figure 14. For $\omega=\omega_{1}$, slightly greater than the critical $\omega_{1 / 3}$, the corresponding response amplitudes are marked by points $R$ and $S$. At $\omega=\omega_{2}$, the response amplitudes are equal and marked by point $T$.

To illustrate the idea of the control, recall that the response curves depend on the forcing amplitude $F$ and the phase $\phi$ (see Figure 13 in the case of harmonic responses). Suppose that we have a set of response curves for $F=F_{0}$ and $\phi=0$. For a fixed frequency $\omega=\omega_{1 / 3}$ under small perturbation, the point $P$ will move to a neighboring $P^{\prime}$, at which the subharmonic no longer exists. Therefore the system responds purely at the forcing harmonic. At a greater frequency $\omega=\omega_{1}$ $>\omega_{1 / 3}$, as $F_{1} \rightarrow \mathrm{F}$ and $\phi \neq 0$, a likely scenario is for the shift of the solid curves in Figure 14 to the dashed curves as depicted in Figure 15. Note, at the same frequency $\omega=\omega_{l}$, the point $R$ disappears and $S$ moves to $S^{\prime}$ as the new (dashed) curve for the harmonic response after the control was applied to the amplitude 
$F$ and the phase $\phi$ in the deriving force. Figure 15 shows the basic idea of control for the Duffing equation (1).

\section{Conclusion}

Nonlinear time series analysis is used to explore complex phenomena and uncover underlying patterns from irregular data from experiments. Power spectral density analyses were performed for three unrelated experiments and their nonlinear behaviors were captured; then the response was changed to a lower stable state, maintaining near conservation of energy. We have examined the control by using a small adjustment by feedback, utilized a method to track the origin of the information, and established the role of the initial conditions on the control strategy.

Results are on the control of shock waves from an axisymmetric supersonic jet, control of periodic waves on a vibrating panel forced by turbulent boundary layer flow and sound, and control of a subharmonic and harmonic of a panel forced by sound. Control is achieved by using the individual initial conditions. In all three experiments, control was achieved by the distribution of power over the spectral band. As a result, the fundamental and harmonic spectral peaks reduce to flat broadband level responses in the first two experiments, whereas the subharmonic and harmonic responses are shifted and added to the fundamental in the third experiment.

This paper is a study of time-evolving processes; stability is a central issue, which has revolutionized our understanding of these processes. They contribute to a new vista of applications close to conservation or no loss of energy. Conservation rests on the experimental fact that the responses of flow, structure, and sound are inherently unstable; the dynamics processes are associated with energy transfer over the experiment time and are applicable to technological problems in a wide range of fields. This paper started from observing data of interest, and analysis and methodology were developed to illuminate properties of the origin of the data.

\section{References}

1. Ott, E., Grebogi, C., and Yorke, J. A., "Controlling Chaos," Phys. Rev. Lett., Vol. 64, pp. 1196-1999, 1990.
2. Shinbrot, T., Grebogi, C., Ot, E., and Yorke, J. A., "Using Small Perturbations to Control Chaos," Nature, Vol. 363, pp. 411-417, June 1993.

3. Wehrmann, O., "Reduction of Velocity Fluctuation in a Karman Vortex Street by a Vibrating Cylinder," Phys. Fluids, Vol. 8, pp. 760-761, 1965.

4. Ditto, W. L., Rauseo, S. N., and Spano, M. L. "Experimental Control of Chaos," Phys. Rev. Lett., Vol. 65, pp. 3211-3214, 1990.

5. Hubiger, B., Doemer, R., Heng, H., and Marienssen, W., "Approaching Nonlinear Dynamics by Studying the Motion of a Pendulum, III. Predictability and Chaotic Motion," Intl. J. Bifur. Chaos in Appl. Sci. Eng., Vol. 4, No. 4, pp. 773-784, 1994.

6. Schwartz, I. B., and Triandaf, I., "Tracking Unstable Orbits in Experiments.," Phys. Rev. A: Gen. Phys., Vol. 46, pp. 7439-7444, 1992.

7. Pyragas: K., and Tamasevicius, A., "Experimental Control of Chaos by Delayed Self-Controlling Feedback," Phys. Lett. A, Vol. 180, pp. 99-102, 1993.

8. Chow, P. L., and Maestrello, L., "Stabilization of Non-Linear Panel Vibration by Boundary Layer Damping," J. Sound Vib., Vol. 182, No. 4, pp. 541-558, 1995.

9. Maestrello, L., "Controlling Chaos via Knowledge of Initial Condition for a Curved Structure," AIAA Paper 2000-2016, June 2000.

10. Dowell, E. H., "Flutter of a Buckled Plate as an Example of Chaotic Motion of Deterministic Autonomous System." J. Sound Vib., Vol. 85, pp. $334-344,1982$.

11. Dowell, F. H., and Pezeshki, C., "On the Understanding of Chaos in Duffing Equation Including a Comparison With Experiment," Appl. Mech., Vol. 53, No. 1, pp. 5-9, 1986.

12. Mei, C., Abdel-Motagaly, K., and Chen, R., "Review of Nonlinear Panel Flutter at Supersonic and Hypersonic Speeds," ASME Appl. Mech. Rev., Vol. 52, No. 10, pp. 321-332, Oct. 1999.

13. Maestrello, L., "Control of Shock Loading From a Jet in the Presence of a Flexible Structure," AIAA J., Vol. 38, 2000. To be published. 
14. Maestrello, L., "Active Control of Panel Oscillation Induced by Accelerated Turbulent Boundary Layer and Sound," AIAA J., Vol. 35, No. 5. pp. 796-801, 1997.

15. Maestrello, Lucio, "Active Control of Nonlinear Vibration of a Panel Structure," J. Acolist. Soc. Am., Vol. 93, No. 4, Pt. 2, April 1993.

16. Abarbanel, H. D. I., and Sushchik, M. M., "True Lyapunov Exponents Models of Chaotic Data," Intl. J. Bifur. Chaos in Appl. Sci. Eng., Vol. 3, pp. 543-550, 1993.

17. Eckmann, J. P., and Ruelle, D., "Ergotic Theory of Chaos and Strange Attractors," Rev. Mod. Phys., Vol. 57, pp. 617-656, 1985.

18. Parlitz, U., "Identification of True and Spurious Lyapunov Exponents From Time Series," Intl. $J$. Bifur. Chaos in Appl. Sci. Eng., Vol. 2, pp. 155-165, 1992.

19. Maestrello, L., "Chaotic Response of Panel Vibrations Forced by Turbulent Boundary Layer and Sound," AIAA J., Vol. 37, No. 3, pp. 289-295, 1999 ,

20. Arecchi, F. T., Boccaletti, S., Ciofini, M., and Meucci, R., "The Control of Chaos: Theoretical Schemes and Experimental Realizations," Intl. J. Bifur. Chaos in Appl. Sci. Eng., Vol. 8, pp. 1643-1655, 1998.

21. Barenblatt, G. I., "On a Model of LaminarTurbulent Transition," J. Fluid Mech., Vol. 212, pp. 487-496, 1990.

22. Lauterborn, W., and Cramer, E., "Subharmonic Route to Chaos Observed in Acoustics," Phys. Rev. Lett., Vol. 47, pp. 1445-1448, 1981.

23. Maestrello, L., "Control of NonlinearNonstationary Structure Response and Radiation Near a Supersonic Jet," AIAA J., Vol. 32, No. 7, pp. 1367-1376, July 1994.

24. Maestrello, L., Frendi, A., and Brown, D. E., "Nonlinear Vibration and Radiation From a Panel With Transition to Chaos," AIAA J., Vol. 30, No. 11, pp. 2632-2638, Nov. 1992.
25. Powell, A., "On the Mechanism of Choked Jet Noise," Proc. Phys. Soc. London, Vol. PE12, pp. 1039-1056, 1953.

26. Westley, R., and Woolley, J. H., "The Near Field Sound Pressures of a Choked Jet When Oscillating in the Spinning Mode," AIAA Paper 75-479, 1975.

27. Norum, T. D., "Screech Suppression Supersonic Jets," AIAA J., Vol. 21, No. 2, pp. 235-240, 1983.

28. Seiner, J. M., "Advances in High Speed Jet Aeroacoustics,” AIAA Paper 84-2275, Oct. 1984.

29. Ponton, M. K., and Seiner, J. M., "Large Scale Structures of Axisymmetric Supersonic Flow," Proceeding of the Workshop on Nonstationary Stochastic Processes and Their Applications, World Scientific, Singapore, pp. 38-47, 1991.

30. Tam, C. R. W., "The Shock-Cell Structures and Screech Tone Frequencies of Rectangular and Non-Axisymmetric Supersonic Jets," J. Sound Vib., Vol. 121, No. 1, pp. 135-147, 1988.

31. Morris, P. J., Bhat, T. R. S., and Chen, G., "A Linear Shock-Cell Model for Jet of Arbitrary Exit Geometry," J. Sound Vib., Vol. 132, pp. 199-211, 1989.

32. Holmes, C., and Holmes, P., "Second Order Averaging and Bifurcations to Subharmonics in Duffing's Equation," J. Sound Vib., Vol. 78, No. 2, pp. 161-174, 1981 .

33. Krylov, N. M., and Bogoliubov, N. N., "Introduction to Nonlinear Mechanics," Princeton University Press, Princeton, 1947.

34. Iwaya, Y., Suzuki, Y., Sakata, M., and Sone, T., "Active Control of Vibration Intensity in a Beam in Fairfield," J. Acoust. Soc. Japan (e), Vol. 20, No. 5, pp. 347-352, 1999.

35. Stoker, J. J. "Nonlinear Vibrations in Mechanical and Electrical Systems," John Wiley \& Sons, Inc., New York, 1992. 


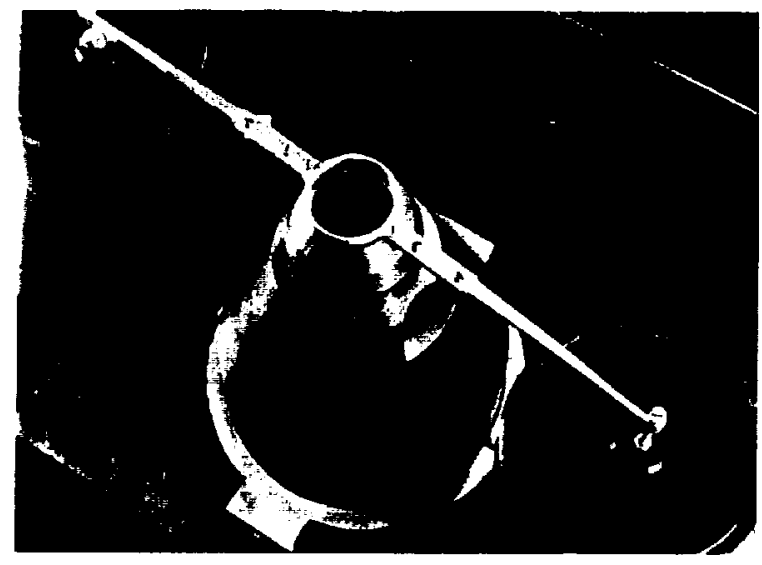

Figure 1. Standard round nozzle with oscillating lip.

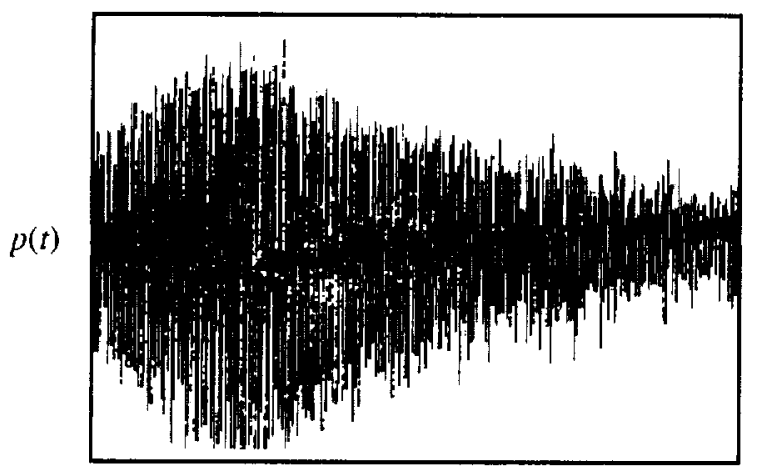

$t, \sec$

Figure 2. Pressure about nozzle exit for uncontrolled run.

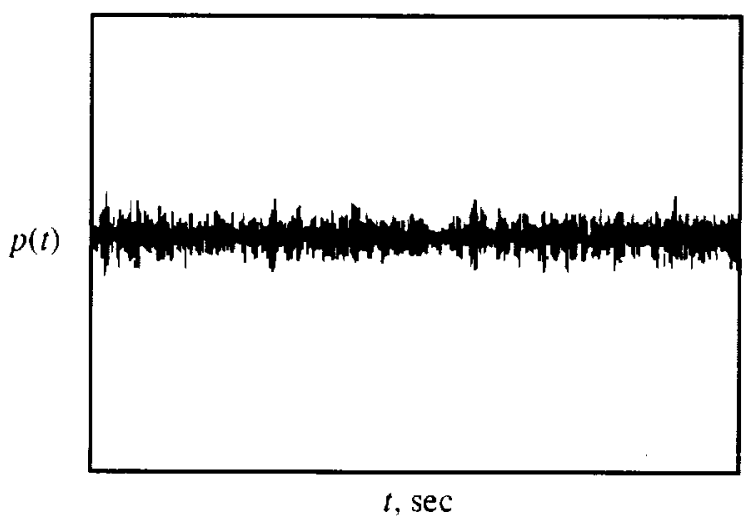

Figure 3. Pressure about nozzle exit for controlled run.

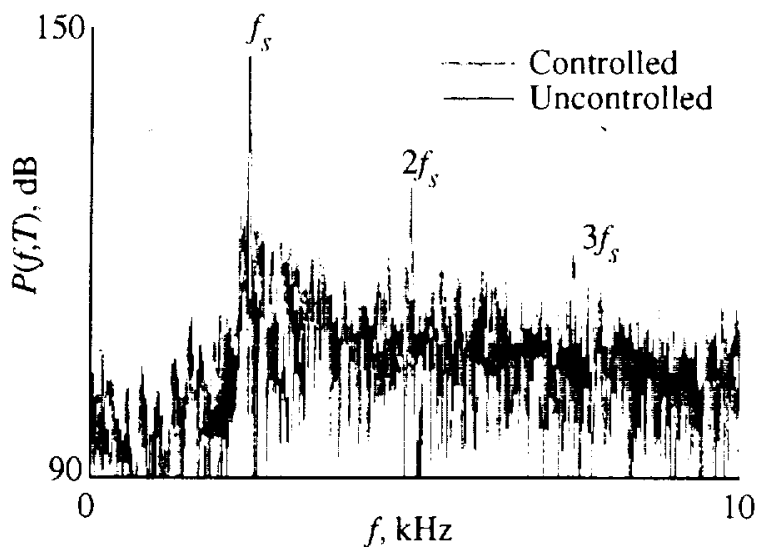

Figure 4. Power spectral density of pressure for uncontrolled and controlled runs.

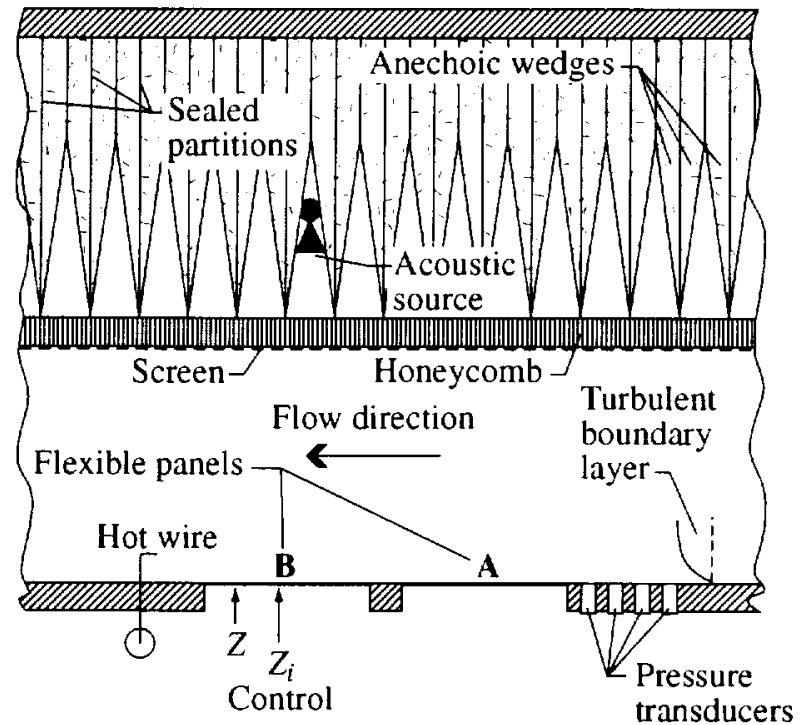

Figure 5. Top view of wind tunnel setup with anechoic test section.

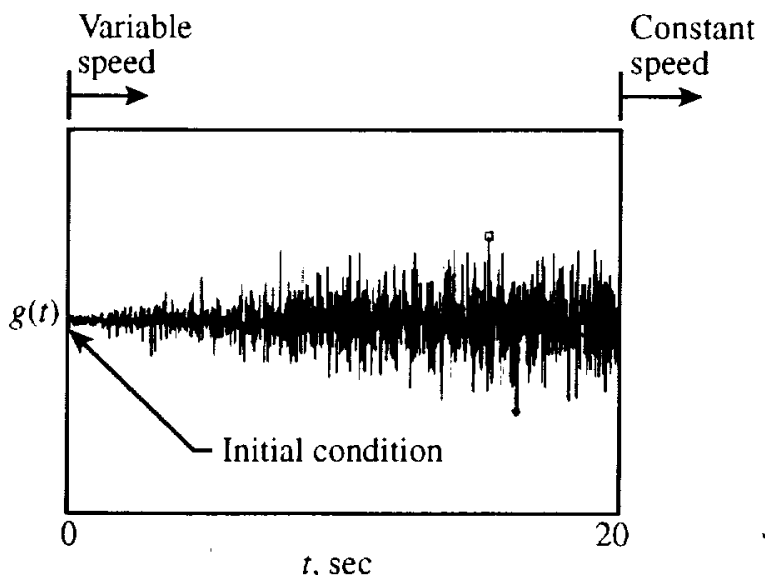

Figure 6. Panel response from turbulent boundary layer loading from state at rest to constant speed. 


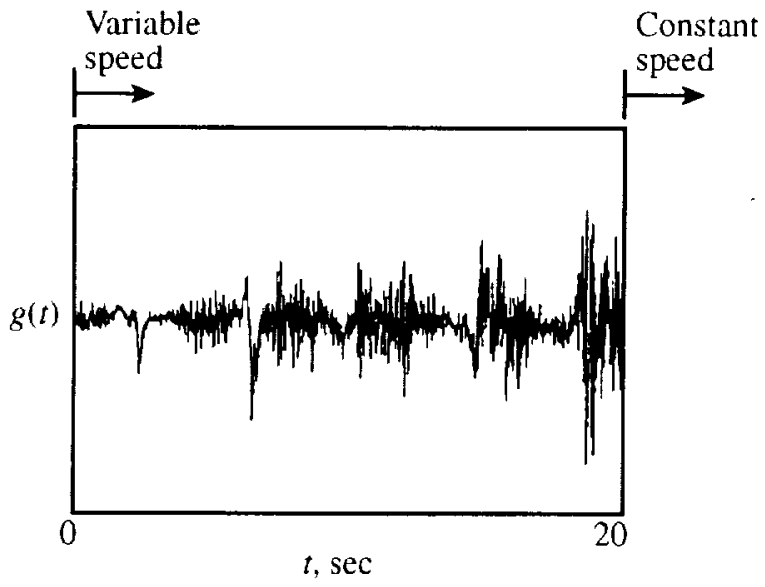

Figure 7. Panel response from turbulent boundary layer and sound loading from state at rest to constant speed

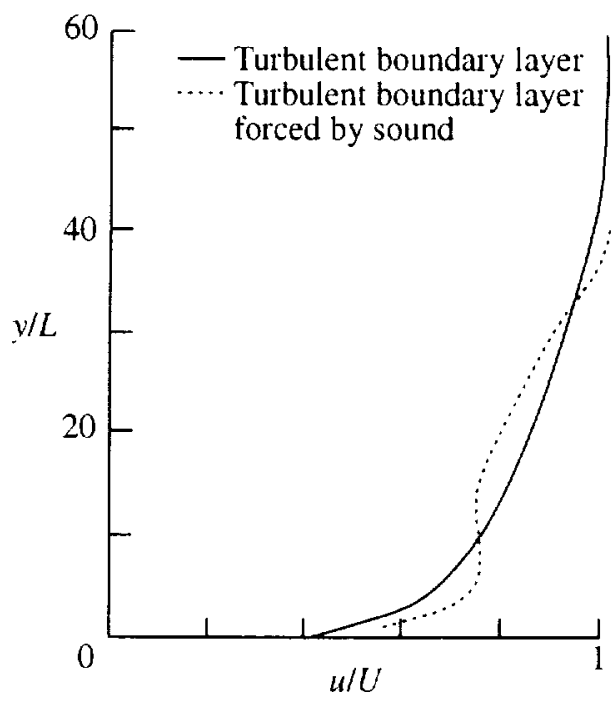

Figure 8 . Velocity profiles of constant speed condition of downstream edge of flexible panel.

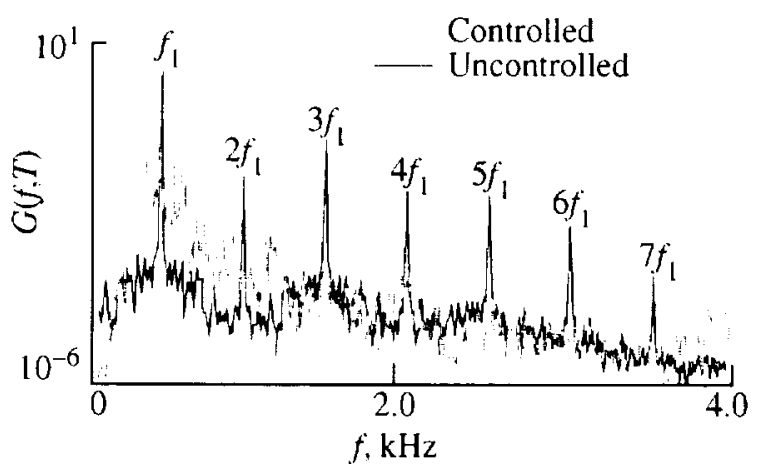

Figure 9. Control of panel response forced by turbulent boundary layer and sound.

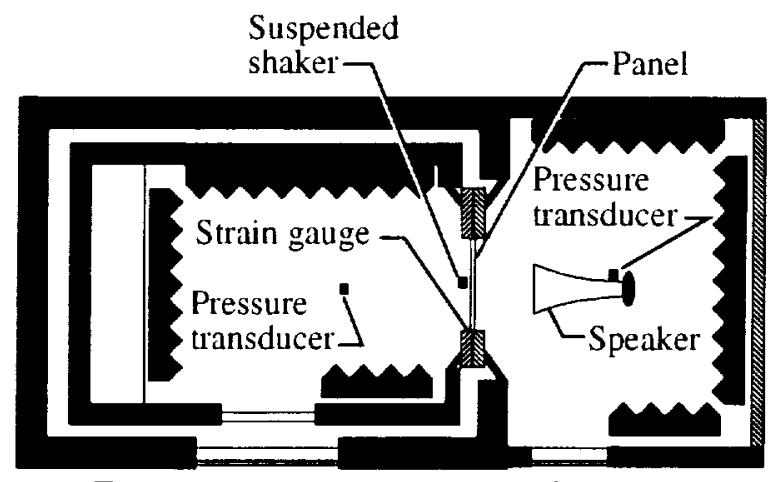

Transmission room

Source room

Figure 10. Experimental setup inside anechoic chambers.

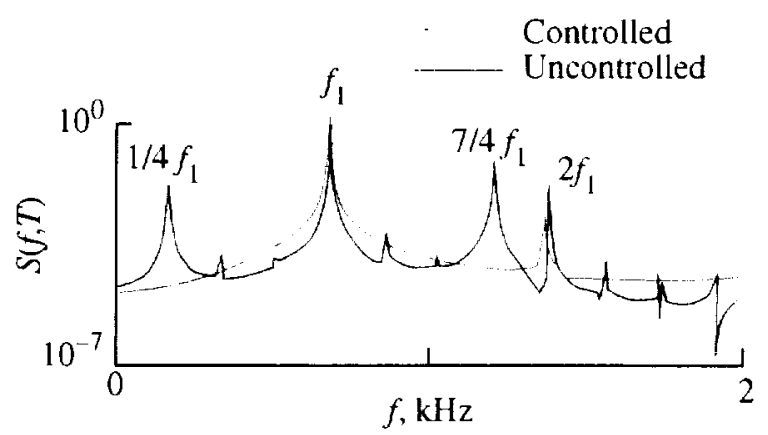

Figure 11. Control of subharmonic and harmonic strain of panel forced by sound.

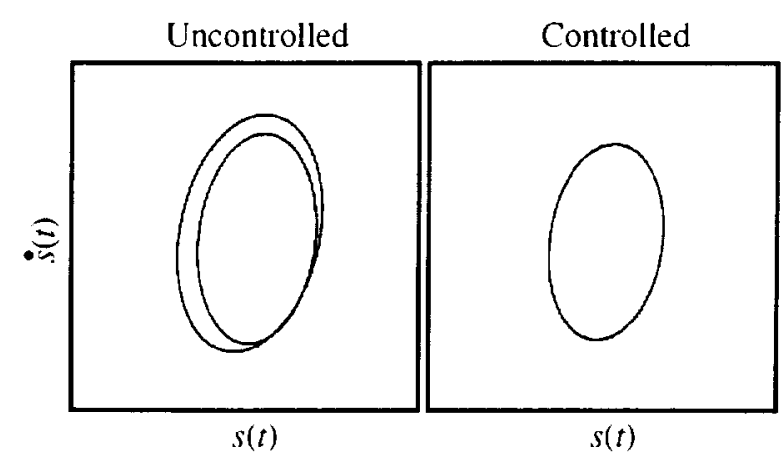

Figure 12. Uncontrolled and controlled panel phase. 


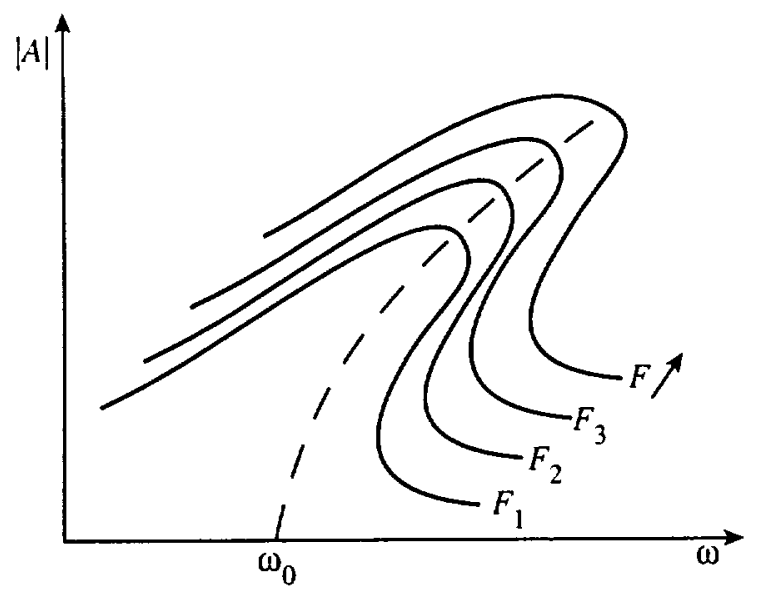

Figure 13. Frequency response curves for harmonic response.

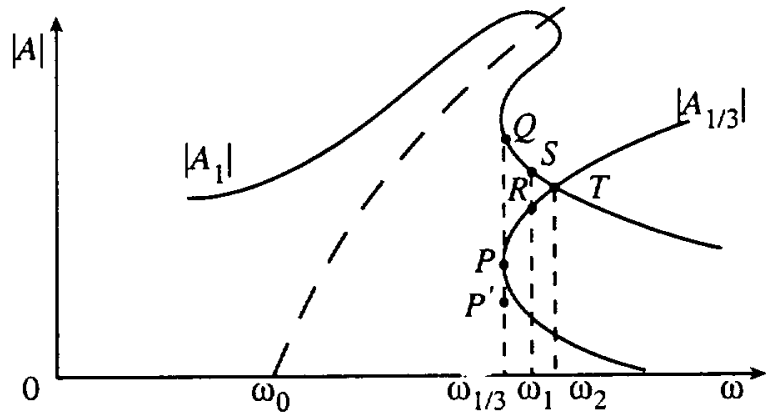

Figure 14. Frequency response curves for harmonic and subharmonic responses.

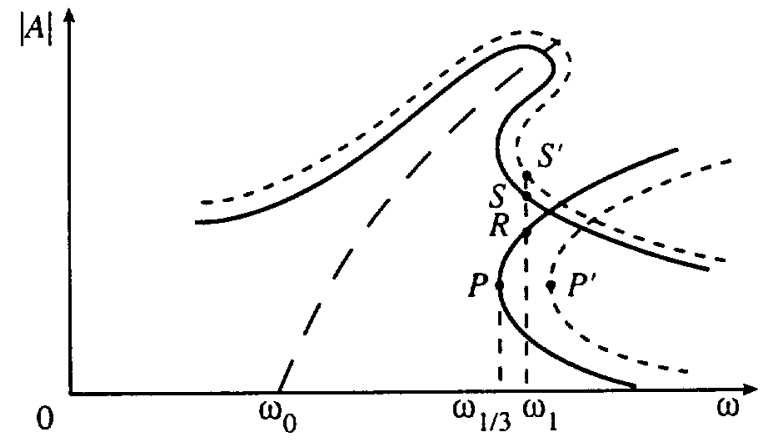

Figure 15. Shift of response curves due to modulation of amplitude $F$ and phase $\phi$. 